\title{
Los gerentes no familiares en las Pequeñas y Medianas Empresas familiares ${ }^{1}$
}

Non-family Managers in Family Small and medium firms Os gerentes não familiares nas PME familiares

Lucila Mariel Garavaglia Università Politecnica delle Marche, Ancona, Italia.

E-mail:garavaglialucila@gmail.com

\author{
Luca Del Bene
}

Università degli Studi di Pisa, Italia.

E-mail:I.delbene@univpm.it

\section{Resumen}

Fecha de recepción: 28/04/2017 Fecha de aceptación: 23/06/2017
Palabras clave - empresas familiares - gerente no familiar - administración
Este trabajo tiene como objetivo profundizar el estudio de la relación existente entre el/los gerentes no familiares y la familia propietaria en la pequeña y mediana empresa familiar. Se trata de un tema de profundo y creciente interés tanto en el mundo académico como empresarial.

Mediante el desarrollo de una investigación cualitativa, se busca comprender el motivo por el cual la empresa familiar decide incorporarlos, cuál es proceso de reclutamiento y selección que realizan, qué actividades desarrollan, y qué tipo de relación mantienen con la familia propietaria. El trabajo de campo se desarrolló en diez PyMEs familiares argentinas e italianas, mediante el desarrollo de entrevistas semi-estructuradas con los gerentes $\mathrm{y} / 0$ directores de dichas empresas.

Los principales resultados señalan una amplia variedad de causas que llevan a contratar gerentes no familiares, lo cual se relaciona a su vez con la gran amplitud de tareas que desarrollan. Además, se destaca un proceso de reclutamiento y selección bastante informal, con una participación activa del emprendedor familiar. Por último, los resultados demuestran la estricta relación laboral que mantienen las partes. 
Keywords

- family businesses - non-family managers

- administration

Palavras-chave

- empresas familiares

- gerente não familiar

- administração

\section{Abstract}

The aim of this article is to delveinto the relationship between non-family managers and owners in family-ownedSMEs. It is a subject of increasing interest in both the academic and business world.

This paper focuses on understanding, through qualitative research, the reason why a family business decides to incorporate a non-family manager, which recruitment and selection processes are followed, the kind of activities developed by these managers, and the type of relationship mantained with the family. The fieldwork was carriedout in ten Argentinian and Italian family-ownedSMEs, through semi-structured interviews to managers and/or directors of such companies.

According to the main results, there is a wide variety of causes for hiring non-family managers, and they are asked to do several tasks of different kinds. In addition, it has been found that the recruitment and selection processes are quite informal, with an active participation of the familymember entrepreneur. Finally, the results show a strict employment relationship between the parties.

\section{Resumo}

Este trabalho tem por objetivo aprofundar no estudo da relação existente entre o/os gerentes não familiares e a família proprietária na pequena e média empresa familiar. Trata-se de um tema de profundo e crescente interesse tanto do mundo acadêmico quanto do empresarial.

Através do desenvolvimento de uma pesquisa qualitativa procura-se compreender o motivo pelo qual a empresa familiar decide incorporá-los, qual é o processo de recrutamento e seleção que realizam, que atividades desenvolvem, e que tipos de relacionamento mantêm com a família proprietária. 0 trabalho de campo desenvolveu-se em dez PME familiares Argentinas e Italianas, por meio de entrevistas semi-estruturadas com os gerentes e/ou diretores das citadas empresas.

Os principais resultados indicam uma ampla variedade de causas que levam a contratar gerentes não familiares, 0 que se relaciona pela sua vez com a grande amplitude de tarefas que desenvolvem. Aliás, destacase um processo de recrutamento e seleção bastante informal, com uma participação ativa do empreendedor familiar. Finalmente, os resultados demonstram a estrita relação laboral que mantêm as partes. 


\section{Introducción}

Los gerentes ${ }^{2}$ no familiares poseen una importancia trascendental en los órganos de administración de las empresas familiares (KPGM, 2014; Del Bene, 2008; Gallo, 1995; Chrisman, Chua y Sharma, 1998). Sin embargo, solo en los últimos años diferentes estudios académicos (Vandekerkhof, Steijvers, Hendriks y Voordeckers, 2014; Dekker, Lybaert, Steijvers y Depaire, 2015; Hiebl, 2013) han focalizado su atención sobre este grupo de profesionales que desarrollan su trabajo entre el mundo de la empresa y de la familia.

Entre los temas más desarrollados por la literatura se encuentran: las posibles ventajas y desventajas que poseen los gerentes familiares en contraposición a aquellos que no lo son (Aronoff y Ward, 1992; Ward, 1997; Chirico, 2008); la relación existente entre el ingreso de uno 0 más managers no familiares a la empresa familiar y los resultados sobre la performance de la misma (Khurana y Nohria, 2000; Bennedsen et ál., 2007; Sciascia y Mazzola, 2008; Miller et ál., 2014; Cucculelli, Mannarino, Pupo y Ricotta, 2014; Dekker et ál., 2015); y la manera en la cual se remunera e incentiva su trabajo (McConaughy, 2000; Gomez-Mejia, Larraza-Kintana y Makri, 2003; Block, 2011; Rodriguez Ariza, Watkins y Briano Turrent, 2015). No obstante, la literatura actual carece de una atención focalizada sobre la relación existente entre la familia propietaria y el/ los gerentes no familiares que se desempeñan en la empresa (Compagno, 2008; Chua, Chrisman y Sharma, 2003). La mayoría de los estudios que han desarrollado el tema lo han hecho en forma secundaria al argumento principal de sus investigaciones (Sonfield y Lussier, 2009).
A su vez, la participación de managers no familiares dentro del órgano de administración es una característica cada vez más común en las empresas familiares de todo el mundo (Block, 2011; Vandekerkhof et ál., 2014; Cerdán, 2010) y genera una enorme preocupación por parte de los fundadores/ CEOs de estas empresas, quienes deben afrontar un proceso nuevo, desconocido y lleno de incertezas. A modo de ejemplo, teniendo en cuenta los resultados de una investigación realizada por Chua J.H., Chrisman J. y Sharma P. (2003) en California, ${ }^{3}$ el segundo problema más importante que afrontan los dirigentes de empresas familiares, según su propia opinión, es la relación con los gerentes no familiares, fundamentalmente cuando se incrementa el tamaño de la empresa y las actividades que desarrollan estos managers resultan vitales para su buen funcionamiento. La principal preocupación continúa siendo la transición en el liderazgo de la empresa familiar, la cual, a su vez, puede presentarse en forma conjunta a la incorporación de un manager externo en la empresa. ${ }^{4}$ Por último, si bien en relación con el principal tema de preocupación existe una vasta literatura disponible, las diversas publicaciones focalizadas sobre los gerentes no familiares en las empresas familiares son escasas, dando origen a una nueva línea de investigación dentro del management de la empresa familiar (Huybrechts, Voordeckers y Lybaert, 2012; Chua et ál., 2003).

La falta de interés por parte de los estudiosos hacia el comportamiento de los managers externos en la empresa familiar puede deberse, entre otras cosas, a la resistencia que generalmente demuestran estas empresas al momento de incorporar miembros no familiares a uno de los órganos de

(2) En este artículo se utilizarán los términos gerente y manager como sinónimos.

(3) Los resultados de la investigación se basan sobre 272 respuestas válidas que fueron obtenidas mediante el cumplimento de un cuestionario enviado a 1725 dirigentes de empresas familiares de diferentes sectores y dimensiones.

(4) Ward (2004) detalla los tres problemas fundamentales que deben afrontar las empresas familiares: en primer lugar se encuentra el proceso de transición emprenditorial, la segunda preocupación es cómo financiar el crecimiento de la empresa y la liquidez de la familia al mismo tiempo, y finalmente, en tercer lugar se encuentra cómo atraer, motivar, compensar y retener a los managers no familiares. 
mayor responsabilidad de la organización. La familia suele inclinarse hacia la intimidad, evitando pedir asesoramiento a miembros que no pertenezcan a ella. En 1992, solo el cinco por ciento de las empresas familiares medianas contaban con al menos un gerente no familiar (Aronoff y Ward, 1992). En los últimos años este número se viene incrementado sostenidamente, principalmente como consecuencia de la creciente complejidad de las actividades y procesos que desarrollan las organizaciones y la rapidez de los cambios que se producen en el mercado. Si bien las estadísticas disponibles son escasas, tomando en cuenta una investigación realizada por KPMG (2014) en 125 empresas familiares de diferentes países, cerca de la mitad de las empresas manifestaron contar con al menos el cincuenta por ciento de su órgano de administración compuesto por gerentes externos. ${ }^{5}$

El objetivo de este trabajo es profundizar el estudio sobre las condiciones bajo las cuales las pequeñas y medianas empresas familiares deciden contratar al menos un gerente no familiar. Mediante el desarrollo de una investigación cualitativa se buscará dar respuesta a las siguientes preguntas: ¿Cuál es el motivo por el cual la empresa familiar decide incorporar uno o más managers externos?, ¿cómo se produce el reclutamiento y la selección de los mismos?, ¿qué actividades desarrollan? y ¿cómo es su relación con los miembros de la familia propietaria?
El presente artículo se encuentra estructurado del siguiente modo: en el segundo punto se desarrollará el análisis de la literatura existente y se propondrán las hipótesis de trabajo; el punto siguiente estará destinado a la metodología de la investigación y a la presentación de las empresas analizadas; el cuarto punto tiene como objetivo la presentación de los resultados de la investigación, y finalmente, el último punto expone las conclusiones del trabajo junto a algunas posibles áreas de estudio para futuras investigaciones.

\section{Revisión de la literatura en relación con los gerentes no familiares y desarrollo de las hipótesis}

\begin{abstract}
¿Qué se entiende por manager externo?
Los managers ${ }^{6}$ externos son personas claves dentro de las empresas familiares que desean crecer y permanecer en el mercado (Dyer, 1989). En algunos casos, la relación existente entre estas personas y la/s familia/s para las cuales trabajan es tan profunda que son considerados «como parte de la familia» (Klein y Bell, 2007; Blumentritt, Keyt y Astrachan, 2007).

Gran parte del desarrollo de la literatura en relación con este argumento se ha llevado a cabo sin definir con exactitud qué se entiende por
\end{abstract}

(5) En otra investigación llevada a cabo por Del Bene (2008) en 184 empresas familiares que transitan al menos la segunda generación y pertenecen a la industria manufacturera italiana, el $29.34 \%$ manifestó emplear managers externos profesionales.

Estos datos son similares a los resultados obtenidos por Cori y Del Bene (2009), donde sobre una muestra de 60 pequeñas y medianas empresas familiares pertenecientes al sector industrial, localizadas en Toscana, Umbria y Le Marche (Italia), y con una fecha de inicio de actividad anterior al 31/12/1980, el $25 \%$ ha declarado la presencia de managers no familiares dentro de la estructura organizativa de la empresa. Por otro lado, Bennedsen et ál. (2007) manifiestan, en base a una investigación realizada en 5334 empresas de Dinamarca, que solo el $33.3 \%$ de las mismas habían seleccionado a un familiar como el futuro sucesor en el liderazgo de la empresa.

Los resultados cambian en función de la edad y dimensión de la empresa familiar. Existe una correlación positiva entre dimensión de la empresa y propensión a la inserción de managers no familiares (McConaughy, 2000; Cori y Del Bene, 2009; Cerdán, 2010; Chrisman, Memili y Misra, 2014; Vandekerkhof et ál., 2014) y entre edad de la empresa y tendencia a la incorporación de managers externos (Cerdán, 2010; Chrisman et ál., 2014).

(6) Managers son las personas encargadas de tomar las decisiones claves de la empresa (Eisenhardt, 1989). 
manager no familiar (Klein y Bell, 2007). Algunos autores hacen referencia al tema utilizando diferentes conceptos tales como: «Ejecutivos/gerentes no familiares», "Key managers» 0 simplemente «managers no familiares». En este trabajo consideraremos manager externo a la persona que se encuentra a cargo de una función o división de la empresa familiar sin ser ni un pariente de sangre de la familia propietaria ni un pariente por matrimonio 0 adopción. A su vez, los términos «manager/ gerente externo» $y$ «manager/gerente no familiar» serán utilizados como sinónimos.

El hecho de considerar un único parámetro a la hora de definir el concepto de manager no familiar genera claridad al momento de determinar quién se encuentra dentro de esta categoría y quién no. Además, permite incluir dentro del concepto a aquellos empleados de la empresa a quienes, ya sea por confianza 0 por el buen desempeño obtenido, se les concede la posibilidad de crecimiento profesional, elevándolos a la categoría de managers de la empresa familiar; así como a aquellos asesores externos que se incorporan a la actividad empresarial con un nuevo cargo como dependientes.

\section{Principales ventajas}

\section{de la incorporación de un gerente externo en la empresa familiar}

Gran parte de los propietarios y directivos de empresas familiares que ya han incorporado al menos un gerente no familiar en la administración de sus negocios sostienen que ha sido una de las mejores decisiones que han tomado (Aronoff y Ward, 1992).

Diferentes autores han dedicado parte de sus trabajos a identificar las potenciales ventajas que la incorporación de un manager externo puede traer (Ward, 1997; Aronoff y Ward, 1992; Chirico, 2008; Sciascia y Mazzola, 2008; Del Bene, Lattanzi y Liberatore, 2012; Di Toma y Montanari, 2013). Entre las principales podemos mencionar:

- Objetividad: un gerente no familiar suele ser la mejor fuente de alimentación (feedback) que una empresa puede tener (Aronoff y Ward, 1992; Chua et ál., 2003). Esto se debe, fundamentalmente, a que el mismo no posee ningún interés personal en la empresa ni es propietario de la misma. Además, un gerente no familiar puede analizar el ambiente organizacional sin el bagaje emocional que implica el hecho de pertenecer a la familia propietaria (Cori y Del Bene, 2008; Fernandez-0rtiz et ál., 2007; Ossorio, 2011).

- Incremento del capital intelectual y social a disposición de la empresa familiar: un manager externo trae consigo nuevos conocimientos, experiencias y competencias que la empresa generalmente necesita para desempeñarse en modo eficiente en el mercado (Dyer, 1989; Ward, 1997; Miller, Steier y Le Breton-Miller, 2003; Chrisman y McMullan, 2004; Lutz y Schraml, 2011; Fernandez-0rtiz et ál., 2007; Cori y Del Bene, 2008; Vandekerkhof et ál., 2014; Perry, Ring y Broberg, 2014). La heterogeneidad en las capacidades de los managers con distintos bagajes de conocimientos y experiencias puede llevar a la toma de decisiones bien informadas y balanceadas (Lutz y Schraml, 2011). Dos investigaciones realizadas por Bennedsen et ál. (2007) y Jorrissen et ál. (2005) señalan que, en promedio, los gerentes no familiares se encuentran más instruidos que los familiares. ${ }^{7}$

(7) No obstante, en una investigación llevada a cabo en México en 69 empresas familiares no financieras cotizadas en la BMV durante el periodo 2001-2012, los CEOs familiares contaban con una mejor preparación académica en comparación con los Directores externos. Se han enfocado más en programas de negocios, han alcanzado niveles académicos superiores, se han graduado en universidades de mayor prestigio y poseen mayor cantidad de años de experiencia dentro de la empresa familiar. Sin embargo, este mayor nivel de educación no se ve reflejado en un mayor rendimiento financiero de las empresas dirigidas por CEOs familiares (Rodriguez Ariza et ál., 2015). 
- Aclaración de los roles a desempeñar: esta ventaja se refiere a dos cuestiones puntuales. Por un lado, a veces los familiares que desempeñan al mismo tiempo un rol de propietario y de miembro del órgano de administración de la empresa, pueden confundir sus diversas responsabilidades en la empresa. En estas situaciones, los managers no familiares son los responsables de recomendarles en cada caso qué posición deben tomar. Por otro lado, en las empresas familiares que han superado la primera generación, los familiares relacionados con el negocio suelen ser varios (propietarios y no), y los gerentes externos son los responsables de generar la fuerza necesaria que permita arribar a un consenso entre los diferentes intereses en juego. A su vez, protegen el equilibrio necesario para el desarrollo de las actividades y la continuidad de la empresa (Bammens, Voordeckers y Van Gils, 2008; Songini y Vola, 2015).

- Profesionalización: algunos estudiosos sostienen que los managers no familiares que se incorporan a la empresa familiar traen consigo disciplina y profesionalización a las diferentes actividades y procesos que se desarrollan dentro de la organización (Dyer, 1989; Dekker et ál., 2015; Songini y Vola, 2015). Con su incorporación generalmente se crean sistemas de control, procedimientos para el logro de objetivos, etc. Estos managers suelen contribuir a optimizar el funcionamiento de las diferentes reuniones y juntas que posee la empresa, mejorando los medios de comunicación, los procedimientos para la toma de decisiones y la calidad de las mismas.

- Disminución del costo marginal: la combinación de propiedad y management familiar posee diversos costos potenciales (Bloom y Van Reenen, 2006).
Seleccionar a los managers teniendo en cuenta solo a los familiares crea un fuerte desincentivo para aquellos empleados no familiares, quienes carecerán de un motivo para capacitarse y mejorar ya que, al menos dentro de la empresa, no poseen posibilidades de crecimiento.

- Apertura empresarial: la incorporación de al menos un manager externo a la empresa familiar es una clara señal de apertura empresarial, tanto para los empleados de la empresa, como para el resto de las partes interesadas (stakeholders). Es un hecho que demuestra que la empresa familiar se encuentra abierta a nuevas ideas (Miller et ál., 2003). Además, mejora la credibilidad de la empresa frente a todos los agentes económicos con los cuales se encuentra relacionada (FernandezOrtiz et ál., 2007).

No obstante todas estas ventajas, la importancia de la incorporación de al menos un gerente no familiar al órgano de administración de la empresa familiar es un fenómeno que ha adquirido reciente importancia. Diez años atrás eran muy pocas las empresas que decidían incorporar estos profesionales dentro de sus estructuras. ${ }^{8}$ Entre las razones de la reticencia de las empresas a la incorporación de un manager no familiar podemos mencionar: algunos fundadores/CEOs de empresas familiares dudan que sus empresas sean lo suficientemente atrayentes para que un profesional externo y con los conocimientos y experiencias necesarias, desee incorporarse; otros se sienten sobrepasados de trabajo y manifiestan no contar con el tiempo necesario para seleccionar y contratar un manager no familiar; 0 temen a la pérdida de poder y control que tal acontecimiento

(8) Tomando en consideración los resultados de una investigación realizada por Cromie et ál. (1995) en 382 empresas de Inglaterra e Irlanda, el $91 \%$ de las empresas familiares y el $79 \%$ de aquellas que no lo eran, no poseían managers externos dentro de sus órganos de administración. Además, más del $70 \%$ de las empresas poseían una estructura de management invariable desde hace más de 8 años, y la cantidad media de miembros en el órgano de administración era en el $90 \%$ de los casos inferior a 5 personas.

También Gersick (1997) ha señalado la poca cantidad de managers no familiares que poseen las empresas. 
les podría ocasionar; ${ }^{9}$ y otros simplemente aún no se lo han propuesto (Aronoff y Ward,1992; Cromie, Stephenson y Monteith,1995).

Por otro lado, si bien no existe un consenso internacional sobre el impacto que la incorporación de al menos un gerente externo puede ocasionar sobre el rendimiento de la empresa (Dekker et ál., 2015; Rodriguez Azira et ál., 2015; Miller y Breton-Miller, 2006; Durendez et ál.,2007; Chua et ál., 2003), la investigación desarrollada por Sciascia y Mazzola (2008) en 620 empresas familiares italianas, la mayoría de pequeña y mediana dimensión, ha demostrado la existencia de una relación negativa entre la participación de la familia en la dirección de la empresa familiar y el rendimiento obtenido por la empresa. En otras palabras, los resultados señalan que el rendimiento disminuye a medida que la cantidad de familiares que se encuentran en las tareas de gerenciamiento de la empresa aumenta. ${ }^{10}$ Estos resultados han llevado a los autores a sugerir la apertura de uno de los órganos responsables de tomar las decisiones estratégicas de la empresa, sobre todo cuando el nivel de participación de la familia es elevado.

Además de esto, Ward (1997) subraya como una de las mejores prácticas que puede llevar a cabo una empresa familiar que desea crecer «la atracción y retención de managers no familiares de excelencia». Curiosamente, las empresas familiares piden consejos a diferentes asesores externos para resolver problemas cotidianos y estratégicos de la empresa, pero encuentran un freno al momento de contratarlos como miembros del órgano de adminis- tración de la sociedad. En ese momento, aprender a delegar y compartir el proceso decisorio se convierte en el principal desafío que la empresa familiar debe afrontar (Cromie et ál., 1995).

Por último, algunos autores consideran el ingreso de managers externos a la empresa familiar como una práctica poco difusa y focalizada en las grandes empresas familiares que cotizan en el mercado financiero (Di Toma y Montanari, 2013).

\section{¿Cuándo y por qué las empresas familiares deciden contratar managers externos?}

El empleo de gerentes externos ayuda a las empresas familiares a incrementar la base de conocimientos, experiencias y competencias que le permitan individuar y/0 crear nuevas oportunidades de negocios (Chirico, 2008; Block, 2011; Bertoldi y Corsico, 2015). No obstante la importancia que poseen los managers no familiares en el interior de la empresa familiar, poco se sabe sobre el momento y el/los motivo/s que llevan a la empresa a contratarlos (Vandekerhof et ál., 2014).

A partir del análisis de la literatura se puede inferir que gran parte de las incorporaciones de managers externos al órgano de administración de la empresa familiar surge como consecuencia del desarrollo y el crecimiento del negocio.

Durante los primeros años de existencia, las empresas familiares son dirigidas y administradas por el/los fundador/es (Gallo, 1998; Gersick, 1997). La estructura de gestión empresarial es bastante informal y el poder de decisión se encuentra concentrado en las manos del fundador y de algunos pocos

\footnotetext{
(9) Chrisman et ál. (2013) manifiestan que la incorporación de al menos un manager no familiar a la empresa implica, muchas veces, renunciar a obtener algunos de los objetivos no económicos de la empresa familiar, como por ejemplo: mantener el control familiar sobre el management, preservar la salud socioemotiva de la familia, tener un comportamiento altruista hacia los miembros de la familia, ete.

Cori y Del Bene (2009) señalan, en base a los resultados de la investigación ya citada, los posibles riesgos que puede traer la inserción de managers no familiares a la empresa: en primer lugar se encuentra la persecución de objetivos personales por parte de los mismos (en vez que empresariales), seguido por la falta de integración con los miembros de la familia, y los posibles conflictos de intereses entre ambos grupos. (10) Resultados similares fueron obtenidos por Dekker et ál. (2015) y Durendez et ál. (2007).
} 
familiares, quienes participan en forma directa del desarrollo de las principales actividades. Esta estructura suele funcionar bastante bien durante los primeros años de la empresa. Un fundador motivado y trabajador es, casi siempre, la principal razón de éxito de una empresa familiar en esta fase (Gedajlovic, Lubatkin y Schulze, 2004).

Cuando la empresa crece en dimensión y sus operaciones de negocios adquieren mayor complejidad, resulta necesario contar con una estructura de gestión más formal, un proceso de descentralización en la toma de decisiones y un órgano de administración que se encuentre lo suficientemente calificado para afrontar la complejidad de la actividad empresarial. Con el paso del tiempo, empresa y familia crecen y se diversifican, y los órganos de dirección y administración son los encargados de velar por la continuidad del negocio. En estas circunstancias, contar con dirigentes capaces, competentes y responsables con su trabajo es un elemento clave para el éxito de la empresa familiar. Klein (2000) y McConaughy (2000) observan que la importancia de los gerentes no familiares se incrementa al aumentar el tamaño de la empresa. ${ }^{11}$ Chirsman y McMullan (2004) al estudiar la performance de las pequeñas y medianas empresas que reciben asesoramiento externo subrayaron que, generalmente, la incorporación de un manager no familiar se lleva a cabo cuando existe una brecha (gap) entre los conocimientos que posee la empresa familiar y aquellos que son necesarios para obtener una performance de éxito.

Cuando el crecimiento empresarial implica la expansión de la organización en sectores/actividades diversas a las originarias, la necesidad de contar con gerentes externos se siente aún más. ${ }^{12}$ Esto se debe a que los managers externos poseen conocimientos y competencias que pueden ser aplicados a numerosas actividades, mientras que los familiares que trabajan en la empresa suelen poseer competencias idiosincráticas y, por lo tanto, utilizables casi exclusivamente en el sector de originario de actividad (Ossorio, 2011). Vandekerkhof et ál. (2014) encontraron una relación positiva entre el nivel de innovación e internacionalización de la empresa familiar y la incorporación de managers no familiares en el equipo de alta dirección (top management team) de la empresa.

Una parte importante del desarrollo de la literatura sobre el comportamiento de los gerentes externos en las empresas familiares y sobre su relación con la familia propietaria fue desarrollada como un subtema dentro del proceso de profesionalización de la empresa familiar. ${ }^{13}$ Si bien ningún autor ha establecido una serie de pasos a adoptar «uno a uno» al momento de decidir profesionalizar la empresa familiar, la incorporación de un manager externo, con los conocimientos y competencias necesarias que le permitan comenzar a realizar el proceso de transición hacia una gestión profesional, puede ser un buen punto de partida. ${ }^{14}$

(11) También Huybrechts et ál. (2012) y Vandekerkhof et ál. (2014) han encontrado una relación significativamente positiva entre el tamaño de la empresa y la presencia de al menos un manager no familiar. La primera investigación se realizó en 740 empresas de Bélgica (de las cuales 555 eran familiares), mientras que la segunda utilizó una muestra de 145 medianas empresas familiares belgas.

(12) Bennedsen et ál, (2007) establece como una de las posibles causas que llevan a incorporar un gerente no familiar, el incremento de las actividades de la empresa, ya sea en volumen como en complejidad.

(13) Sonfield y Lussier (2009) sostienen que el incremento en el número de managers externos en las empresas familiares se encuentra directamente relacionado con el aumento en el uso de asesoría externa especializada, «sophisticated financial management», y con un mayor tiempo de dedicación al desarrollo de estrategias de management.

(14) Del Bene et ál. (2012) definen el término profesionalización como «contratar personas no pertenecientes a la familia con particulares facultades/capacidades de management», y luego agregan que «la profesionalización hace referencia a diferentes aspectos que comprenden: la creación de una estructura managerial, la proyección de diversos mecanismos dirigidos a planificar y controlar el desarrollo de las actividades realizadas, la incorporación de profesionales adecuados al cumplimiento de los objetivos, y la formalización de los instrumentos típicos de la actividad managerial, ya sea desde el punto de vista organizativo como informático». 
En este punto es importante resaltar, como lo hacen Hall y Nordqvist (2008) y Dekker et ál. (2015), la importancia de no confundir los términos «profesionalización»e «incorporación de managers no familiares», ya que el primer concepto es mucho más amplio y puede incluir muchas otras prácticas tales como: la incorporación de sistemas formales de control, la delegación y descentralización de autoridad, cambios en el proceso decisorio, etcétera.

Otros autores relacionan el ingreso de un gerente externo con la necesidad por parte de la empresa familiar de cubrir un puesto clave para el cual no cuentan con ningún familiar que reúna los requisitos necesarios (Dyer, 1989; Berenbeim, 1990; Cromie et ál., 1995), y al mismo tiempo, la empresa tampoco cuenta con el tiempo necesario como para realizar la capacitación de alguno de los familiares para esta función. Esta situación puede presentarse, por ejemplo, de frente al retiro inminente del fundador/ CEO de la empresa. Dentro del proceso sucesorio, la incorporación de un manager no familiar puede realizarse con dos objetivos diversos: como futuro sucesor del emprendedor actual, ${ }^{15} 0$ como apoyo a la nueva generación que ingresa en el negocio (Le Breton-Miller et ál., 2004; Lutz y Schraml, 2011). ${ }^{16}$

Al mismo tiempo, el ingreso de al menos un gerente no familiar puede ser parte de una solución dirigida a resolver los problemas del nepotismo que, algunas veces, limita el crecimiento de la empresa familiar (Dyer, 1989; Di Toma y Montanari, 2013). En las empresas familiares, la probabilidad de divergencia en las opiniones aumenta a medida que se incorporan nuevas generaciones (Ward y Anonoff, 1994). En estos casos, el profesional externo suele intervenir como mediador entre los diferentes intereses y expectativas de los socios y familiares implicados, con el fin de evitar que surjan conflictos destructivos. Generalmente, su actividad se focaliza en proporcionar consejo y control en el desarrollo de las diferentes actividades (Bammens et ál., 2008).

Por otro lado, cuando la empresa crece en tamaño y edad, puede que la familia propietaria ya no desee dedicarse activamente a la empresa, y prefiera confiar en un manager no familiar que cuente con los conocimientos y experiencias necesarias (Hielb, 2013). Generalmente, la familia que decide contratar un manager externo busca despojar de familiaridad y emociones al equipo de gestión (team managment) y construir un proceso decisorio con un estilo más formal (Klein y Bell, 2007).

Todo esto demuestra que, casi siempre, la incorporación de al menos un manager no familiar se produce como consecuencia de una limitación en los recursos humanos disponibles dentro de la familia para afrontar una determinada situación (Chirico, 2008). Esta situación puede ser tanto una crisis, en el ámbito familiar y/o empresarial, como una oportunidad (Klein y Bell, 2007). Según Bennedsen et ál. (2007) las empresas familiares son más propensas a incorporar gerentes no familiares cuando la empresa sufre un shock negativo. Al respecto, no existe un análisis profundo del tema dentro de la literatura actual, la cual solo se limita a mencionar varias situaciones que requieren la incorporación de un manager no familiar.

Como regla, los propietarios de empresas familiares son reacios a ceder — aún parcialmente — el nivel de control que poseen de la empresa, a menos que la continuidad de la misma se encuentre en juego (Gedajlovic et ál., 2004). Esto se debe a que, el ingreso de al menos un manager no familiar puede disminuir la riqueza socioemociona $1{ }^{17}$ de cada

(15) Esta situación podría darse por un período breve, como manager externo «puente» entre dos generaciones con una amplia diferencia de edad y/o formación, que impide el desarrollo de una transición directa entre ambas (Gallo, 1998).

(16) Los propietarios de la empresa familiar pueden incluso estar interesados en contratar un manager externo para que actúe como puente o intermediario entre dos familias propietarias, o entre dos familiares que luchan por alcanzar la posición de máxima responsabilidad de la empresa.

(17) Para mayor detalle sobre este concepto, véase: Gomez-Mejia et ál. (2007) y Gomez-Mejia et ál. (2011). 
propietario (ese conjunto de beneficios no financieros como: la posibilidad de ejercer la autoridad, de perpetuar los valores de la familia, y de ser altruista, entre los principales), sin embargo, en ocasiones ésta puede ser la única opción para mantener la empresa en funcionamiento (Huybrechts et ál., 2012). Contratar un manager externo, delegarle responsabilidades, sobre una estructura de comando independiente de la familia, son todas acciones que probablemente disminuirán el poder de control sobre las decisiones estratégicas de la empresa (GomezMejia, Cruz, Berrone y De Castro, 2011). Esto, a su vez, incrementará los conflictos sobre los objetivos como consecuencia de las motivaciones e intereses divergentes entre los empleados familiares y quienes no lo son (Gersick, 1997).

A partir de este análisis surgen las primeras hipótesis de este estudio:

Hipótesis 1: La incorporación de al menos un manager externo a la empresa familiar es un acontecimiento que se desarrolla luego de la creación y despegue de la empresa.

Esto quiere decir que las pequeñas y medianas empresas familiares nacen y comienzan a desarrollar sus actividades sin contar con la presencia de un manager no familiar en su órgano de administración. El ingreso de los mismos se produce con el paso del tiempo, generalmente luego de la etapa de despegue del negocio.

Hipótesis 2: El principal motivo que lleva a la empresa familiar a incorporar un gerente externo tiene que ver con salvaguardar la continuidad del negocio, de frente a una situación de riesgo presente o futura.

\section{¿Quién elige a los gerentes no familiares? y ¿qué criterios se tienen en cuenta?}

Una de las áreas de estudio menos desarrolladas en relación con la incorporación de al menos un manager externo a la empresa familiar tiene que ver con el proceso de reclutamiento, selección y retención del mismo (Klein y Bell, 2007; Block, 2011). ¿Cuáles son los principales criterios a tener en cuenta? Por ejemplo, al momento de asignar una determinada prioridad, ies más importante contar con un MBA (Master in Business Administration), tener experiencia en otras empresas familiares o poseer una cultura y valores similares a los de la empresa familiar?

Sin lugar a duda, la selección de un manager externo es uno de los mayores desafíos que la empresa familiar debe afrontar (Klein y Bell, 2007; Bertoldi y Corsico, 2015). Algunas veces, las empresas familiares con poca o ninguna experiencia en el proceso de reclutamiento y selección de managers, tienden a focalizar su atención sobre la experiencia profesional del candidato, haciendo énfasis en los antecedentes que posea en el área en el cual la empresa tenga las mayores dificultades, sin tomar en cuenta las posibles diferencias culturales entre ambos, y el proceso de adaptación mutua que se requiere (Hiebl, 2013; Hall y Nordqvist, 2008). Esta situación podría provocar futuras incomprensiones y conflictos entre los managers familiares y aquellos que no lo son (Hall y Nordqvist, 2008).

Si bien el nivel de educación formal que un candidato posee es un requisito muy importante (el candidato «ideal» debería tener profundos conocimientos en el área de gerenciamiento y debería haber adquirido experiencia y competencias en el área/sector en el cual se desarrolla la empresa familiar), por sí solo no basta a la hora de seleccionar el mejor candidato a ocupar el puesto de gerenciamiento. Es esencial tener en cuenta el contexto social y cultural de cada persona y evaluar las posibilidades de entendimiento mutuo que existen entre el candidato, los managers familiares y el resto de las personas que se desempeñan en la empresa. La semejanza de valores, normas, objetivos y cultura suele ser un aspecto olvidado o secundario, tanto para la empresa familiar que desea incorporar un nuevo profesional, como para el candidato que se postula al cargo. Sin embargo, es un aspecto vital 
que impacta directamente sobre el futuro entendimiento mutuo de las partes (Hall y Nordqvist, 2008; Blumentritt et ál., 2007; Block, 2011; Bertoldi y Corsico, 2015).

Tanto el carácter como la integridad de los candidatos a managers no familiares son aspectos fundamentales a tener en cuenta en el proceso decisorio. Estos requisitos, a menudo, poseen una mayor incidencia en las empresas familiares, en comparación a aquellas que no lo son (Blumentritt et ál., 2007). El carácter es esencial para trabajar en forma eficiente dentro del contexto de una empresa familiar. A su vez, los gerentes no familiares de éxito utilizan sus competencias para influenciar sobre los aspectos principales de la empresa familiar, con el objetivo de salvaguardar la continuidad de la misma. Durante los períodos de crisis, en los cuales estos managers suelen ingresar a la empresa, es fundamental contar con las competencias y actitudes necesarias que permitan generar un punto de equilibrio/consenso entre los intereses de la familia, no siempre unificados, y los de la empresa.

Otro aspecto poco analizado por la literatura actual, y relacionado con el tema antes desarrollado, es la importancia que posee la confianza dentro del proceso de selección de un manager no familiar. Cabe hacernos la siguiente pregunta: ¿Las empresas que deciden incorporar un manager no familiar, dan prioridad a sus empleados, 0 a alguno de sus asesores, o se arriesgan a incorporar un profesional con el cual no han tenido ninguna experiencia previa? Resulta claro que, las personas que poseen cierta relación con la empresa familiar cuentan con la ventaja de conocer, al menos en parte, su cultura, valores e intereses, pero quizás, en esta ocasión la empresa privilegie la incorpora- ción de nuevos aires en el interior de la estructura empresarial. ${ }^{18}$

Esto nos lleva a la tercera hipótesis:

Hipótesis 3: En el proceso de reclutamiento y selección de managers no familiares, la empresa busca siempre primero de ascender a algunos de sus empleados antes de dirigirse al mercado laboral externo para encontrar un candidato.

Volviendo sobre cuáles son los principales requisitos que el candidato a manager no familiar debe satisfacer, los resultados obtenidos por Cori y Del Bene (2009) señalan que las características más importantes que el empresario busca en el gerente no familiar son: en primer lugar un elevado conocimiento de las competencias técnicas necesarias, seguido muy de cerca por la capacidad relacional. Así mismo, la confianza mutua y la coherencia entre los valores de la empresa familiar y los del candidato poseen una importancia relevante.

Bandiera, Guiso, Prat y Sadun, (2008) agruparon los diferentes criterios a tener en cuenta al momento de contratar un manager no familiar en dos modelos:

- El modelo del desempeño (performance): donde los managers son contratados en base al desempeño esperado, analizando los antecedentes disponibles (educación, éxito en trabajos anteriores, etc.). Esto es evaluado regularmente en base a criterios predeterminados y medibles. Los gerentes que poseen rendimientos inferiores a los esperados son separados del proceso.

- El modelo de la fidelidad: donde existe, en cambio, un importante componente relacional. Los managers son contratados en base a la fidelidad prevista, por lo

(18) A modo de ejemplo, en la investigación realizada por Cromie et ál. (1995), previamente citada, el 90 $\%$ de las empresas consultadas manifestaron que preferían ascender a un empleado de la empresa antes que contratar un profesional externo para un cargo de alta jerarquía. Estos resultados concuerdan con la opinión de Ossorio (2011) quien sostiene que «en la etapa de selección de los candidatos, será más fácil para la familia evaluar a colaboradores internos antes que a personas provenientes de otras carreras profesionales realizadas fuera de la empresa familiar...La empresa posee la certeza de que los colaboradores internos comparten el sistema de normas y valores que guía la estrategia empresarial». 
tanto, el conocimiento personal directo previo es un factor muy apreciado. Las evaluaciones objetivas del desempeño son poco utilizadas. Los managers son promovidos o desvinculados en función de la calidad de la relación que posean con la familia propietaria.

Naturalmente, ninguna empresa utiliza un modelo puro de desempeño o de fidelidad. Según Bandiera et ál., (2008), en Italia, las empresas familiares y aquellas que operan solo en el mercado nacional son más propensas a inclinarse por el modelo de fidelidad. A su vez, al momento de la elección, estas empresas suelen basarse más en las referencias que poseen de los candidatos, obtenidas tanto por miembros de la empresa como por su red de contactos (socialnetwork), que en encuentros formales de evaluación de las características de cada candidato (GomezMejia et ál., 2011; Bertoldi y Corsico; 2015), y son más propensas a promover un manager que posee una buena relación con la familia propietaria, que uno que realice un buen desempeño.

Hipótesis 4: Al momento de contratar un manager no familiar, la confianza en el candidato es el requisito más importante a evaluar, seguido por las competencias formales (conocimientos y experiencia).

En otras palabras, tener confianza en el nuevo manager es un punto fundamental, y es uno de los principales motivos por los cuales se comienza a realizar el proceso de búsqueda entre los empleados de la empresa. Sin embargo, por si solo este requisito no basta. Las competencias formales también poseen gran importancia y el candidato deberá reunir ambos requisitos.

Existen pocas investigaciones sobre quién debería conducir el proceso de reclutamiento y selección de los manager no familiares. Algunos autores como Astrachan et ál. (2002) y Aronoff y Ward (2000) sugieren que este proceso debería ser confiado a un grupo de personas expertas, independientes, neutrales e imparciales. Por ejemplo, en una investigación realizada por Von Schultzendorff (1984), el 70 $\%$ de los managers consultados afirmaron haber sido seleccionados por personas externas a la empresa, mientras que solo el $30 \%$ sostuvo haber sido seleccionado por miembros de la familia propietaria.

Por último, algunos procesos de selección de gerentes externos en las empresas familiares incorporan generalmente, y forma adicional, una mayor cantidad de elementos informales al proceso de evaluación (Klein y Bell, 2007). Por ejemplo: un encuentro familiar fuera del ámbito de trabajo, el conocimiento de toda la familia del futuro manager, etcétera.

A partir de este análisis, la última hipótesis de este trabajo es:

Hipótesis 5: La selección de un gerente no familiar es un proceso que recae principalmente sobre el fundador/emprendedor familiar.

\section{Metodología: el análisis de algunos casos de estudio}

El análisis empírico fue realizado mediate el desarrollo de diez casos de estudio. El trabajo de campo se desarrolló en Le Marche (Italia) y en Santa Fe (Argentina). Los principales motivos que nos llevaron a seleccionar este método de investigación fueron: se trata de un método que permite investigar fenómenos en forma profunda y además posibilita la obtención de diversas perspectivas de cada caso en particular (Yin, 1994; Fattore, 2005). Asimismo, el método de estudio de casos es particularmente apropiado en nuevas áreas de investigación (Eisenhardt, 1989), cuando el tema es contemporáne $0^{19}$ 
(Yin, 1994; Eisenhardt y Graebner, 2007), y cuando el investigador posee poco o ningún control sobre el fenómeno 0 evento estudiado (Yin, 2014). Se trata de un método útil para describir situaciones y eventos complejos y proponer, solo proponer, posibles explicaciones y reflexiones de carácter general (Fattore, 2005). Como sostiene Yin (1994), el estudio de casos múltiples proporciona una base sólida para la construcción teórica.

En relación con el número de casos, si bien no existe un número «ideal», la literatura sugiere entre cuatro y diez casos, hasta llegar al punto de saturación teórica, es decir, al punto en el cual cada caso adicional agrega un aprendizaje adicional mínimo (Eisenhardt, 1989).

Sin embargo, desde el punto de vista teórico y científico, el método de estudio seleccionado presenta ciertas limitaciones que no pueden ser ignoradas. Los principales motivos por los cuales este método es criticado son: a) el método no responde a determinados criterios de rigor científico ya sea, por la falta de un método compartido, la presencia de excesivos espacios de subjetividad en el proceso de análisis e interpretación de los datos, y por la carencia de adecuados instrumentos de control intersubjetivos (Yin, 1994). b) El problema de la generalización de resultados. Se trata de un método difícil de aplicar a un gran número de empresas y, no permite realizar inferencias hacia el exterior de la muestra analizada, convirtiéndolo en un método poco útil para producir conocimientos de carácter general. ${ }^{20} \mathrm{c}$ ) El método no responde al principio de economicidad: por el valor del resultado capaz de producir, utiliza recursos excesivos. d) La atendibilidad de los resultados. ${ }^{21}$ Se trata de un criterio muy difícil de respetar ya que la variedad de los instrumentos de investigación utilizados en los estudios de casos hacen generalmente único el trabajo de investigación.

No obstante estas limitaciones, consideramos que el estudio de casos es el mejor método de investigación disponible para analizar esta problemática ya que existen diversos actores involucrados, se trata de un evento/fenómeno contemporáneo 0 relacionado con un pasado muy reciente, en donde quienes investigan pueden entrar en contacto directo con quienes forman parte del fenómeno bajo estudio. Si bien se analizan una menor cantidad de unidades (en este caso son solo 10 empresas), el grado de profundidad es mayor (se pueden abarcar una mayor cantidad de variables utilizando diversas técnicas de estudio), y cada caso en sí mismo amerita un análisis en profundidad.

La investigación empírica fue realizada en el período comprendido entre Agosto 2015 y Abril 2016, mediante entrevistas de naturaleza semiestructurada, llevadas a cabo con el auxilio de una hoja de ruta/control (checklist) y profundizadas con preguntas específicas y conversaciones informales con el emprendedor actual de cada empresa familiar, o con alguno de sus principales dirigentes. También se entrevistaron dos gerentes generales externos pertenecientes a dos de las empresas italianas que participan del estudio. El principal objetivo de esta investigación es tendencialmente explorativo (Fattore, 2005).

En relación con la selección de los casos, en primer lugar se establecieron ciertos requisitos que la empresa familiar debía satisfacer:

(20) La no generalización de los resultados representa un límite y el precio que el investigador debe pagar por adoptar una estrategia de investigación que permite estudiar un fenómeno en mayor profundidad. Se trata de un método más rico y flexible en cuanto a la información que permite obtener, pero menos controlable en comparación a los estudios cuantitativos.

(21) Según Fattore (2005), la atendibilidad hace referencia a la posibilidad de reproducción de los resultados de las mediciones realizadas. Agrega que la misma señala el grado con el cual la medición realizada permite obtener los mismos resultados en diversas pruebas con el mismo instrumento o con instrumentos equivalentes. 
- Pequeñas y medianas empresas familiares según la definición de la Fundación Observatorio pyme (FOP). ${ }^{22}$

- Al menos la segunda generación debe estar al mando de la empresa.

- Debe poseer al menos un manager no familiar.

- Pertenecientes al sector industrial y/o comercial (bienes y servicios).

El método de muestreo seleccionado fue el de «Bola de nieve». Una vez que se ha corroborado que la empresa reúne los requisitos previamente mencionados, la elección final dependió de la voluntad de cada organización de participar en el estudio.

Los casos analizados se encuentran sintéticamente descriptos en la tabla 1. Con el objetivo de preservar la privacidad de cada empresa analizada se han utilizado letras para distinguir cada empresa familiar entrevistada y se hace referencia al cargo de la persona entrevistada, en lugar de a su nombre.

Tabla 1. Descripción de las empresas analizadas

\begin{tabular}{|c|c|c|c|c|c|}
\hline & EMPRESA & CARACTERISTICAS & $\begin{array}{l}\text { GENERACION } \\
\text { AL MANDO }\end{array}$ & $\begin{array}{l}\text { CANTIDAD DE } \\
\text { GERENTES NO } \\
\text { FAMILIARES } \\
\end{array}$ & $\begin{array}{l}\text { PERSONA } \\
\text { ENTREVISTADA }\end{array}$ \\
\hline 1 & A & $\begin{array}{l}\text { Sector Industrial. Metalúrgica (Arg.) } \\
\text { Empleados: } 160 \\
\text { Antigüedad: } 38 \text { Años (1977) } \\
\end{array}$ & $2^{\circ}$ & 1 & $\begin{array}{l}\text { Gerente General. Miembro } \\
\text { de la segunda generación. }\end{array}$ \\
\hline 2 & B & $\begin{array}{l}\text { Sector comercial. Artículos para el hogar (Arg). } \\
\text { Empleados: } 147 . \\
\text { Antigüedad: } 45 \text { años (1970) } \\
\end{array}$ & $3^{\circ}$ & 2 & $\begin{array}{l}\text { Gerente. Miembro de } \\
\text { la tercera generación. }\end{array}$ \\
\hline 3 & $C$ & $\begin{array}{l}\text { Sector Industrial. Alimentaria (Arg.) } \\
\text { Empleados: } 40 \\
\text { Antigüedad: } 44 \text { años (1971) } \\
\end{array}$ & $2^{\circ}$ & 1 & $\begin{array}{l}\text { Director. Miembro de } \\
\text { la segunda generación. }\end{array}$ \\
\hline 4 & $D$ & $\begin{array}{l}\text { Sector Comercial. Autopartes (Arg). } \\
\text { Empleados: } 68 \\
\text { Antigüedad: } 55 \text { años (1960) } \\
\end{array}$ & $2^{\circ}$ & 4 & $\begin{array}{l}\text { Gerente Genera. Miembro } \\
\text { de la segunda generación. }\end{array}$ \\
\hline 5 & $E$ & $\begin{array}{l}\text { Sector Industrial. Metalúrgica (Arg). } \\
\text { Empleados:165 } \\
\text { Antigüedad: } 31 \text { años (1984) } \\
\end{array}$ & $2^{\circ}$ & 5 & $\begin{array}{l}\text { Gerente General. Miembro } \\
\text { de la segunda generación. }\end{array}$ \\
\hline 6 & $\mathrm{~F}$ & $\begin{array}{l}\text { Sector Servicios. Transporte (Italia) } \\
\text { Empleados: } 56 \\
\text { Antigüedad: } 134 \text { años (1881) }\end{array}$ & $4^{\circ}$ & 1 & $\begin{array}{l}\text { Presidente del Consejo } \\
\text { de Administracion y miembro } \\
\text { de la cuarta generación. }\end{array}$ \\
\hline
\end{tabular}

(22) En el tramo industrial, FOP define como pyme a las empresas que poseen entre 10 y 200 ocupados: son pequeñas si tienen entre 10 y 50 trabajadores y medianas si poseen entre 51 y 200 . En cambio, en el caso de los sectores comercio, servicios a la producción, software y servicios informáticos, y construcción se considera que el tamaño en términos de ocupados es menor, y las pyme son aquellas empresas de entre 5 y 150 empleados. 


\begin{tabular}{|c|c|c|c|c|}
\hline $\begin{array}{l}\text { EMPRESA } \\
\text { [Cont.] }\end{array}$ & CARACTERISTICAS & $\begin{array}{l}\text { GENERACION } \\
\text { AL MANDO }\end{array}$ & $\begin{array}{l}\text { CANTIDAD DE } \\
\text { GERENTES NO } \\
\text { FAMILIARES } \\
\end{array}$ & $\begin{array}{l}\text { PERSONA } \\
\text { ENTREVISTADA }\end{array}$ \\
\hline G & $\begin{array}{l}\text { Sector Industrial. Alimenticia. (Italia) } \\
\text { Empleados: } 40 \\
\text { Antigüedad: } 115 \text { años (1900) }\end{array}$ & $3^{\circ}$ & 2 & $\begin{array}{l}\text { Gerente General. Miembro } \\
\text { de la tercera generación }\end{array}$ \\
\hline$H$ & $\begin{array}{l}\text { Sector Comercial. Distribuidora (Italia) } \\
\text { Empleados: } 150 \\
\text { Antigüedad: } 124 \text { años (1892) }\end{array}$ & $4^{\circ}$ & 1 & $\begin{array}{l}\text { Vicepresidente, miembro } \\
\text { de la cuarta generación, } \\
\text { y al Gerente general externo. }\end{array}$ \\
\hline I & $\begin{array}{l}\text { Sector Industrial. Electrodomésticos (Italia) } \\
\text { Empleados: } 100 \\
\text { Antiguedad: } 79 \text { años (1936) }\end{array}$ & $2^{\circ}$ & 2 & $\begin{array}{l}\text { Gerente general. Miembro } \\
\text { de la segunda generación. }\end{array}$ \\
\hline J & $\begin{array}{l}\text { Sector industrial.Indumentaria. (Italia) } \\
\text { Empleados: } 152 \\
\text { Antigüedad: } 53 \text { años (1963) }\end{array}$ & $3^{\circ}$ & 3 & $\begin{array}{l}\text { Presidente y miembro } \\
\text { de la segunda generación, } \\
\text { y al gerente general externo. }\end{array}$ \\
\hline
\end{tabular}

Fuente: Elaboración propia.

Como se evidencia en la Tabla 1, las empresas familiares que participaron de las entrevistas pertenecen a diversos sectores, cuentan con diferentes tamaños y años de trayectoria. El objeto fue obtener diferentes perspectivas y experiencias sobre la incorporación de managers externos a la vida de la empresa familiar.

Las entrevistas fueron registradas y transcriptas. Cada entrevista junto al resto de la información secundaria aportada por la empresa (revistas, libros de historias familiares, información publicada en el sitio web y en diferentes noticias, etc) fue analizada siguiendo un procedimiento en común, explorando los mismos puntos/características de cada empresa. Luego se realizó un análisis cruzado de casos para identificar las posibles semejanzas y diferencias entre los casos analizados. Cada entrevista fue releída varias veces y cada dato se analizó bajo diversas perspectivas buscando la mayor objetividad posible.

La duración promedio de las entrevistas realizadas fue de entre 45 y 60 minutos. Las preguntas estuvieron focalizadas sobre 5 temas: el momento y las causas que llevaron a la empresa a contratar un manager no familiar, quiénes fueron los respon- sables del proceso de reclutamiento y selección, qué criterios utilizaron para tomar la decisión, qué actividades desarrolla el manager dentro de la empresa en los diferentes órganos que la componen y qué tipo de relación posee con la familia propietaria.

\section{Resultados}

La primera conclusión que se puede extraer en relación con el momento de incorporación del primer gerente no familiar a la actividad empresarial es que se trata de un evento posterior al nacimiento y desarrollo de la empresa familiar (confirma la hipótesis $N^{\circ} 1$ ). Si bien el método de investigación elegido no nos permite hacer inferencias hacia el exterior de los casos estudiados, todas las empresas encuestadas incorporaron el primer manager no familiar luego de al menos diez años de actividad. En el caso de las empresas italianas, si bien en promedio poseen una mayor antigüedad que las empresas argentinas entrevistadas, ninguna incorporó el primer gerente no familiar mientras el fundador estaba en la empresa. 
La contemporaneidad del evento es un punto que no puede ser marginado. Nueve de las diez empresas estudiadas manifestaron haber realizado la primera incorporación de un gerente no familiar a partir del año 1990. Como ya lo habían señalado algunos autores como Aronoff y Ward (1992) y Cromie et ál. (1995), se trata de un acontecimiento de reciente y creciente importancia, que se manifiesta como consecuencia de la diversidad y complejidad de las actividades que deben desarrollar las empresas para mantenerse competitivas en el mercado. A su vez, en los últimos diez años la mitad de las empresas analizadas continuaron incorporando nuevos managers no familiares a la actividad empresarial.

En relación con el motivo de la incorporación, sin lugar a duda la sensación de "amenaza» hacia la continuidad de la empresa es el vínculo conector (hipótesis N² confirmada). Esta «amenaza» puede tener diferentes orígenes que resumimos en dos líneas principales:

En primer lugar está el aumento, en parte imprevisto, de la actividad de la empresa, tanto en cantidad como en complejidad, lo cual genera la necesidad de cambio. Cinco de las diez empresas analizadas declararon haber realizado la primera incorporación de un gerente no familiar con el objetivo de poder afrontar un período de crecimiento y de grandes cambios que tenían de frente. El incremento de la complejidad del ambiente y de la empresa, sumado al crecimiento de la competencia como consecuencia de la globalización del mercado, creaba la necesidad de nuevos conocimientos y perspectivas que las empresas no poseían. Estas empresas, si bien eran conscientes de la necesidad del cambio, no sabían cómo afrontarlo con la estructura que tenían y decidieron recurrir a gerentes no familiares que les sirvan de sostén. Algunas de las empresas entrevistadas protagonizaron un gran desarrollo en pocos años, y los managers no familiares se convirtieron en los encargados de alcanzar gran parte de los objetivos que el fundador y su familia no podían lograr, ya sea por falta de capacidades, exceso de trabajo en otras áreas, o una combinación de ambos factores. Esta perspectiva concuerda con lo manifestado por Klein (2000), Chirsman y McMullan (2004) y Chirico (2008), entre otros.

La segunda línea hace referencia a la necesidad de cubrir un puesto clave dentro de la estructura de la empresa familiar. Se lo considera una amenaza ya que, generalmente, la empresa pierde a uno de sus recursos humanos más valiosos en forma repentina, y no cuenta con otra persona dentro de la familia que pueda asumir esas responsabilidades, ni tampoco cuenta con el tiempo necesario como para preparar a un miembro de la familia para cubrir dicho puesto. Este motivo coincide con lo expresado por Dyer (1989), Berenbeim (1990) y Cromie et ál., (1995), entre otros, y fue la principal razón por la cual dos de las diez empresas entrevistadas decidieron realizar la primera incorporación de un gerente no familiar. Una empresa italiana recurrió a un manager externo ante la muerte repentina del Gerente General/Presidente de la empresa. Como la generación siguiente aún era muy joven y carecía de la preparación adecuada, el manager externo fue una solución justa que permitió darle continuidad a la empresa y actualmente, luego de casi diez años de actividad, esta persona se encuentra capacitando a la nueva generación de la familia, con la cual realizarán la próxima transición empresarial. Otra de las empresas familiares que formó parte del estudio declaró haber realizado la primera incorporación de un gerente no familiar luego de que uno de los accionistas de la empresa vendiera su parte a otro de los socios y se desvinculara como Director/ responsable de Fabricación e Ingeniería.

Un caso que vale la pena mencionar es el de una empresa familiar italiana en donde la Dirección General, mientras la tercera generación se encontraba al mando de la empresa, estaba dividida en tres áreas, cada una de las cuales estaba a cargo de un hermano. La segunda generación de la empresa había fallecido en forma repentina y al llegar la tercera se dividieron la empresa en diferentes áreas sin que exista un gerente general, sino 
que había tres. Al momento de organizar el siguiente pasaje generacional, los socios y los miembros de la cuarta generación estuvieron de acuerdo en que asignarle a un miembro de la nueva generación la responsabilidad de crear una gerencia general y a su vez convertirse en el nuevo líder de la empresa, era una carga demasiado pesada. Por tal motivo, decidieron ascender a un empleado con una amplia trayectoria tanto en la empresa como fuera de ella, al puesto de Gerente General, y años más tarde realizar una nueva transición entre este gerente y la nueva generación. Según la opinión de uno de los miembros de la cuarta generación: «Ninguno de los tres jóvenes que formamos parte de la cuarta generación teníamos ganas de vivir semejante desafío ya que a nivel profesional era muy difícil, pero a nivel humano simbolizaba «escalar el Everest». Entonces encontramos en el Doctor.... la persona que reunía todas las características y a su vez contaba con la espalda lo suficientemente grande como para sentarse en un consejo de administración con cinco familiares pertenecientes a diversas generaciones».

Tanto este como uno de los casos mencionados anteriormente son un claro ejemplo del rol que poseen los manager externos como «puentes» entre dos generaciones con edades y formaciones muy diferentes (Gallo, 1998).

Por último, dos de las empresas bajo análisis decidieron incorporar el primer manager no familiar dentro de un proceso de profesionalización que estaban realizando. Se trata de un motivo que se encuentra directamente vinculado con los anteriores ya que el objetivo de una de las empresas familiares era dejar la empresa lo más ordenada y autónoma posible antes de la llegada de la nueva generación, mientras que la segunda organización había crecido demasiado rápido, necesitaba crear nuevas áreas y contaba con una limitación en el protocolo familiar en cuanto a la cantidad de miembros familiares que pueden trabajar en la empresa.

Según el testimonio del presidente de una de las industrias italianas con más de setenta años en el mercado, antes de la incorporación del primer gerente no familiar, la empresa solía caracterizarse por una organización y gestión más informal y directa, en donde la familia, fundamentalmente aquellas personas que poseían el capital, creían tener derecho para opinar e intervenir en el desarrollo de todas las actividades. Al ser interrogado sobre la dificultad del proceso, el presidente de esta empresa agregó: «El problema no es fácil, es dificilísimo, porque implica el hecho de cambiar la mentalidad de las personas, de lo que podía ser una gestión familiar y directa, a una gestión indirecta en este caso, no es un proceso fácil. No es fácil porque la empresa para funcionar bien, al menos desde la perspectiva como lo vengo analizando en los últimos 10/15 años, debe dividirse y respetar el funcionamiento de sus tres esquemas: el de los accionistas (la propiedad), que debe nominar a los miembros del Consejo de Administración, y a su vez el Consejo de Administración debe encontrar los funcionarios y dirigentes que puedan llevar adelante la empresa. La realización de esta división genera siempre un poco de dificultad, porque los accionistas se sienten un poco desplazados de la actividad que siempre han realizado. Sin embargo, estoy convencido de que este es el camino que todas las pequeñas y medianas empresas deben seguir».

Bennedsen et ál., (2007) sostiene que las empresas familiares son más propensas a incorporar managers no familiares cuando se encuentran de frente a una situación negativa. Esta situación negativa genera una «amenaza» para la empresa familiar, «amenaza» que también suele tener origen en no poder aprovechar una posible situación positiva (crecimiento, diversificación, etc.), por falta de los recursos humanos necesarios. Recién en el momento en el cual la empresa detecta esta "amenaza», y es consciente del riesgo que implica para su continuidad, sus directivos están dispuestos a renunciar a parte de su riqueza socioemocional (Gomez-Mejia et ál., 2007), a cambio de preservar a continuidad y rentabilidad del negocio. Esto coincide con lo sostenido por Huybrechts et ál. (2012), Gomez Mejia et ál. (2011) y Gedajlovic et ál. (2004). 
Los gerentes no familiares suelen tener gran autonomía dentro de su área de especialización (Administración, Finanzas, Ingeniería, etc.), pero siempre dentro una organización donde el fundador 0 emprendedor actual es el centro de la integración. Gallo (1998) los califica como «complemento» para definir su rol dentro de la empresa familiar. En aproximadamente la mitad de los casos analizados, a diferencia de los señalado por Bennedsen et ál. (2007) y Jorrissen et ál. (2005), los gerentes externos no poseían una mayor formación que los gerentes familiares, sino más bien una formación diferente y complementaria con la de los managers familiares.

Al conversar con dos de los managers no familiares contratados por dos de las empresas familiares entrevistadas para desempeñarse como los máximos responsables de la administración de la empresa, ambos resaltaban como una gran ventaja de trabajar en pymes familiares, haber logrado tener autonomía de gestión para tomar las decisiones que les competían, pero siempre contando con el apoyo y consejo de quienes reflejaban la visión de la empresa. Coincidieron en la importancia de sentirse parte de una organización en donde quienes establecen las estrategias a llevar a cabo y los objetivos generales de la empresa se encuentran cercanos y consideran de gran interés el aporte que ellos pueden realizar desde la gestión.

Es importante resaltar que, en la mitad de los casos analizados los managers externos no llegan a formar parte del órgano de dirección de la empresa familiar, lo cual no significa que este órgano sea cien por ciento familiar ya que algunas veces se contratan asesores externos como directores. Solo cuatro de las diez empresas analizadas declararon tener órganos de gobierno compuestos solamente por miembros de la familia. No obstante, esto no significa que los gerentes no familiares no sean incluidos e informados de las razones y circunstancias que afectan o pueden afectar la evolución de la empresa familiar en su estructura de capital y de gobierno, y en su posicionamiento estratégico de crecimiento, diversificación, inversiones y asunción de riesgos. Según el testimonio de los entrevistados, la empresa siempre busca demostrarle al manager no familiar que sus opiniones serán tenidas en cuenta y lealmente consideradas. ${ }^{23}$

En relación con el proceso de reclutamiento y selección de los managers no familiares, todas las empresas entrevistadas manifestaron que en primer lugar intentan promover a alguien interno, y solo en caso de ausencia, recurren al mercado laboral externo (confirma Hipótesis 3). Esta política se realiza no solo para ofrecer una posibilidad de crecimiento y desarrollo a aquellas personas que ya se desempeñan en la empresa, sino también por la importancia que tiene para las empresas familiares, el hecho de compartir ciertos valores, propósitos y la cultura de la organización. Como manifestaba uno de los empresarios argentinos entrevistado: "Lo primero que miro es «la cuna», eso de venir y saludar todos los días, la humildad, después miro que sea «pro», porque obviamente que me interesa eso, pero tuvimos malas experiencias con gente que sabía mucho pero nunca se pudo amalgamar al resto por el trato que tenía. Eran más jefes que líderes, y yo necesito tener más líderes, necesito gente que venga y tenga la empatía.... Que además de saber tenga la humildad de darse con el otro, y que los otros los valoren... y no que venga con la "gineta» por lo que sabe o por lo que vivió. Necesito gente que respete y comparta la cultura y los valores de la empresa». Además, la capacidad de trabajar en equipo en uno de los principales requisitos que las empresas familiares buscan

(23) En cuanto a la conformación del órgano de gobierno, vale la pena resaltar que, en los casos analizados, las empresas que manifestaron tener una composición cien por ciento familiar sostuvieron a su vez que la existencia del órgano de gobierno como tal constituye una mera «formalidad», y que generalmente las principales decisiones de la empresa se toman en modo informal entre los principales miembros de la familia propietaria. 
al momento de contratar un manager externo, y quienes ya se desempeñan en la empresa cuentan con cierta ventaja al conocer a las personas con las cuales deberán trabajar.

Con respecto a la hipótesis $\mathrm{N}^{\circ} 4:$ «Al momento de contratar un gerente no familiar, la confianza en el candidato es el requisito más importante a evaluar, seguido por las competencias formales (conocimientos y experiencia)», nuestro estudio no permite sostenerla, si bien se manifiesta en algunos casos. Tanto la confianza como las competencias formales son requisitos indispensables al momento de contratar un gerente no familiar, pero no existe una preferencia absoluta de un elemento por otro entre las diferentes empresas analizadas, e inclusive dentro de cada empresa a veces se privilegian determinados conocimientos y características y otras veces otros, pero ambos deben estar presentes. Así se manifestaba uno de los Gerentes Generales entrevistados: "Si vos analizás que buscamos siempre promover en primer lugar a alguien interno en la empresa, es porque nos importa mucho la confianza. Ambas cosas, conocimiento y confianza. $Y$ bueno, hay veces que le falta experiencia, pero eso se va haciendo».

Quizás, cuanto mayor dificultad encuentra la empresa en el mercado laboral para conseguir un determinado perfil (sobre todo en cuanto a conocimientos formales específicos), mayor importancia se le da, mientras que cuando existen varios candidatos que reúnen las competencias formales solicitadas por la empresa, el criterio que hace la diferencia es la confianza que cada postulante genera.

En cuanto a quién realiza el proceso de selección, en todos los casos analizados la elección final estuvo a cargo de un miembro de la familia, el emprendedor, algunas veces acompañado por otros familiares y empleados de alta categoría (Confirma hipótesis $\mathrm{N}^{\circ} 5$ ). Es importante resaltar que, en aquellas empresas donde coexisten trabajando dos generaciones de la familia, ambas suelen intervenir en la selección. Por otro lado, seis de las diez empresas estudiadas solicitaron asesora- miento externo para el proceso de reclutamiento de los candidatos a managers no familiares, pero la selección final siempre estuvo a cargo de la familia, y más específicamente del emprendedor. Por último, tres de las empresas afirmaron haber realizado una consulta al Directorio sobre una selección de candidatos posibles al cargo.

Dentro del proceso de reclutamiento y selección de los managers no familiares, las referencias que poseen las empresas de los candidatos tienen un rol clave y forman parte de uno de los principales factores bajo análisis. Esto coincide con lo manifestado por Gomez-Mejia et ál. (2011) y Bertoldi y Corsico (2015) y a su vez es un modo útil para conocer y comprender algunas características del candidato que no se vislumbran en un currículum 0 entrevista, por ejemplo: sus valores, modos de actuar, cultura, etcétera.

Un punto que vale la pena resaltar de las entrevistas realizadas es la falta de mecanismos y procedimientos de control formal que existe en las empresas familiares en relación con el trabajo de los gerentes externos. Solo tres de las diez empresas analizadas manifestaron contar con procedimientos e instrumentos establecidos para evaluar el desempeño de estos managers, el resto sostuvo hacerlo en modo informal y no en forma regular. Quienes cuentan con procesos estandarizados sostienen que es el Directorio de la empresa el órgano encargado de realizar esta labor, mientras que el resto manifestó que son los propios emprendedores quienes evalúan el trabajo de los managers.

En cuanto al tipo de relación que poseen los managers no familiares con la familia propietaria, todas las empresas sostuvieron tener una relación de respeto, cordialidad y profesionalidad, pero sin estrechar vínculos de amistad. Los gerentes no familiares solo se relacionan con algunos de los familiares que trabajan dentro de la empresa, y mantienen una relación laboral. Esto se diferencia con lo manifestado por Klein y Bell (2007) y Blumentritt et ál. (2007) quienes sostenían que los managers no familiares eran considerados muchas 
veces «como parte de la familia». Tener un trato más distante y profesional, según el testimonio de uno de los entrevistados, genera una ventaja para ambas partes: al manager externo le permite ser más objetivo e independiente al momento de tomar una decisión 0 una determinada postura en un problema específico, y a la familia le da mayor «libertad» (sin presiones extras) al momento de señalar comportamientos inapropiados o errores.

\section{Conclusiones}

Este artículo se encargó de analizar la relación existente entre las empresas familiares y los gerentes no familiares. A través de la realización de una investigación cualitativa en diez pymes familiares argentinas e italianas, buscamos dar respuesta a algunos interrogantes como: cuál es el motivo por el cual la empresa familiar decide incorporar a uno 0 más managers externos al desarrollo de su actividad, quién/quienes se encargan del proceso de reclutamiento y selección de los mismos, qué actividades desarrollan y qué relación poseen con la familia propietaria. Elegimos este tema de investigación por dos motivos: porque creemos que el capitalismo familiar tiene una gran necesidad de gerenciamiento (familiar o no), y porque estamos convencidos de las ventajas y la relevancia que implica contar con al menos un manager no familiar dentro de la empresa, sobre todo cuando ésta adquiere cierto tamaño y las nuevas generaciones se van incorporando.

Al finalizar cada una de las entrevistas realizadas, le solicitábamos a los entrevistados que nos den su opinión sobre las ventajas y desventajas que tuvo la incorporación de un gerente externo. Las desventajas fueron nulas en la mayoría de los casos, mientras que algunos manifestaron la dificultad de tomar la decisión y de implementarla en forma eficiente y profesional, pero luego siempre resaltaban que las recompensas fueron mucho mayores: objetividad, independencia, apoyo/mediación, profesionalidad, fueron las principales virtudes destacadas. El Gerente General de una de las empresas familiares entrevistadas sostuvo: "En las pequeñas y medianas empresas los recursos humanos son nuestra principal fuente de riqueza, los managers externos son agentes de cambios, cambios que son necesarios para desarrollarnos y continuar. Son personas objetivas que priorizan la empresa, y lo que la empresa necesita es objetividad».

En relación con los resultados alcanzados, cuatro de las cinco hipótesis elaboradas se han podido confirmar, mientras que la hipótesis $n^{\circ} 4$ no pudo ser sostenida, si bien se vio reflejada en algunos de los casos analizados.

A partir de la investigación realizada pudimos detectar dos nuevas líneas de investigación: a) Por un lado creemos que es necesario profundizar el estudio sobre la composición y el funcionamiento del órgano de gobierno de las empresas familiares. Esto se debe a que varias de las empresas analizadas, a pesar de la trayectoria con la que cuentan, manifestaron no poseer un directorio u organismo equivalente que funcione eficientemente. Para algunas de estas empresas, el órgano de gobierno solo existe en «los papeles» y en el día a día las principales decisiones se toman en modo informal. Sin embargo, a medida que la empresa y la familia crecen y se diversifican, contar con un órgano de gobierno eficaz y eficiente suele ser un punto clave hacia la continuidad; b) Por otro lado, consideramos importante profundizar el estudio de los diversos mecanismos de control que se pueden implementar para vigilar la actividad que desarrollan los managers no familiares. Esto responde a una clara necesidad de las empresas, manifestada por gran parte de los encuestados, y a su vez a una ausencia de instrumentos y mecanismos en los casos analizados.

Deseamos que esta investigación sea de gran utilidad para todos aquellos propietarios y directivos de empresas familiares, que se encuentran analizando la posibilidad de incorporar profesionales externos a la familia, así como también para 
aquellos managers no familiares que ya se desempeñan en diversas empresas o que desean hacerlo, para que puedan comprender las principales dificultades, desafíos y oportunidades que les esperan. A su vez, creemos que los resultados alcanzados pueden ser útiles a toda la comunidad científica, ya que profundizan una problemática contemporánea, ofreciendo diversas perspectivas y soluciones para cada uno de los temas estudiados, y además se detallan nuevas áreas posibles de estudio para continuar contribuyendo al desarrollo y continuidad de las pymes familiares.

\section{Referencias bibliográficas}

- Aronoff, C.E., y Ward, J.L. (1992). Outside Directors: How they help you. Nation's Business, 80(10), 62-64.

- Aronoff, C., y Ward, J. (2000). Family business values: How to assure a legacy of continuity and success. Family business leadership series, 12. Marietta, GA: Business Owner Resources.

- Astrachan, J., Keyt, A., Lane, S., y Yarmalouk, D. (2002). Non-family CEOS in the family business: Connecting family values to business success. En M. Koiranen y N. Karlsson (Eds.), Proceedings from the 2002 Family Business Network Research Forum. Helsinki, Finland: Tampere-paino 0y.

- Bammens, Y., Voordeckers, W., y Van Gils, A. (2008). Boards of directors in family firms: A generational perspective. Small Business Economics, 31(2), 163-180.

- Bandiera, 0., Guiso, L., Prat, A., y Sadun, R. (2008). Italian managers: Fidelity or performance. Ro- dolfo De Benedetti Foundation Working Paper. Recuperado en Julio 2017 de http://www.ceriba.org.uk/ pub/Colnvest/COINVESTCostevent/ rapporto_2_may_14.pdf

- Bennedsen, M., Nielsen, K.M., Perez-Gonzalez, F., y Wolfenson, D. (2007, mayo). Inside the family firm: the role of families in successions decisions and performance. The Quarterly Journal of Ecomonics, 647-691.

- Berenbeim, R.E. (1990). How business families manage the transition from owner to professional management. Family Business Review, 3(1), 69-110.

- Bertoldi, B., y Corsico, F. (2015). Manager di famiglia: come I manager vengono scelti e hanno successo nel capitalism familiare. Italia: Gruppo II Sole 24 ore.

- Block, J.H. (2011). How to pay Nonfamily Managers in Large Family Firms: A principal-agent model. Fam- ily Business Review, 24(1), 9-27.

- Bloom, N., y Van Reenen, J. (2006). Measuring and explaining management practices across firms and countries. National Bureau of Economic Research. CEP Discussion paper 716. Recuperado en Julio 2017 de http://cep.Ise.ac.uk/pubs/download/dp0716.pdf

- Blumentritt, T.P., Keyt, A.D., y Astrachan, J.H. (2007). Creating an environment for successful nonfamily CEOs: An exploratory study of good principals. Family Business Review, 20(4), 321-335.

- Cerdán, Á.L.M. (2010). Análisis del nivel de profesionalización en la empresa familiar. Revista de Estudios Empresariales. Segunda Época, (2), 80-98.

- Chirico, F. (2008). The creation, sharing and transfer of knowledge in family business. Journal of Small Business \& Entrepreneurship, 21(4), 413-433. 
- Chua, J.H., Chrisman, J.J., y Sharma, P. (2003). Succession and nonsuccession concerns of family firms and agency relationship with nonfamily managers. Family Business Review, 16(2), 89-107.

- Chrisman, J.J., Memili, E., y Misra, K. (2014). Nonfamily Managers, Family Firms, and the Winner's Curse: The Influence of Noneconomic Goals and Bounded Rationality. Entrepreneurship Theory and Practice, 38(5), 1103-1127

- Chrisman, J.J., Sharma, P., Steier, L. P., y Chua, J. H. (2013). The influence of family goals, governance, and resources on firm outcomes. Entrepreneurship Theory and Practice, 37(6), 1249-1261.

- Chrisman, J.J., y McMullan, W.E. (2004). Outsider assistance as a knowledge resource for new venture survival. Journal of Small Business Management, 42(3), 229-244.

- Chrisman, J.J., Chua, J.H., y Sharma, P. (1998). Important attributes of successors in family businesses: An exploratory study. Family business review, 11(1), 19-34.

- Compagno, C. (2008). La ricerca sull'impresa familiare. Stato dell'arte e problemi aperti. En C. Devecchi y E.G. Fraquelli (Eds.), Dinamiche di sviluppo e internazionalizzazione del family business (pp. 78-79). Bologna: il Mulino.

- Cori, E., y Del Bene, L. (2009). Condizioni di efficacia dei processi di professionalizzazione delle PMI familiari: il ruolo delle competenze organizzative. En Marchi, L., Marasca S. (a cura di), Le risorse immateriali nell'economia delle aziende. Bologna: II Mulino.

- Cromie, S., Stephenson, B., y Monteith, D. (1995). The management of family firms: an empirical investigation. International Small Business Journal, 13(4), 11-34.

- Cucculelli, M., Mannarino, L., Pupo, V., y Ricotta, F. (2014). Owner Management, Firm Age, and Productivity in Italian Family Firms. Journal of Small Business Management, 52(2), 325-343.

- Dekker, J., Lybaert, N., Steijvers, T., y Depaire, B. (2015). The effect of family business professionalization as a multidimensional construct on firm performance. Journal of Small Business Management, 53(2), 516-538.

- Del Bene, L. (2008). Processi di professionalizzazione delle imprese familiari. Alcune evidenze empiriche. En G. Fraquelli y C. Devecchi (a cura di), Dinamiche di sviluppo e internazionalizzazione del family business. Bologna: II Mulino.

- Del Bene, L., Lattanzi, N., y Liberatore, G. (2012). Aziende famigliari e longevità economica. Modalità di analisi e strumenti operativi. Milán, Italia: IPSOA.

- Di Toma, P., y Montanari, S. (2013). Corporate Governance nelle Imprese Familiari. Italia: Franco Angeli.

- Duréndez, A., de Lema, D. P., y Guijarro, A. M. (2007). Advantages of professionally managed family firms in Spain. En N. Gupta, L. Levenburg, J. Moore y S. Motwani, Culturallysensitive models of family business in Latin Europe: A compendium using the globe paradigm (pp. 88-113). Hyderabad: ICFAl University Press

- Dyer, W.G. (1989). Integrating professional management into a family owned business. Family Business Review, 2(3), 221-235.

- Eisenhardt, K.M., y Graebner, M.E. (2007). Theory building from cases: opportunities and challenges. Academy of management journal, 50(1), 25-32.

- Eisenhardt, K.M. (1989). Building theories from case study research. Academy of management review, 14(4), 532-550.

- Fattore, G. (2005). Metodologia di ricerca in economia aziendale. Milano: Egea.

- Fernández-Ortiz, R., Rodríguez Osés, J.E., y Corcuera, R.0. (2007). Papel de los outsiders en la empresa familiar. Investigaciones europeas de dirección de la empresa (IEDEE), 13(1), 117-129.

- Gallo, M.Á. (1995). Family businesses in Spain: Tracks followed and outcomes reached by those among the largest thousand. Family Business Review, 8(4), 245-254.

- Gallo, M.Á. (1998). La sucesión en la empresa familiar. Colección de Estudios e informes, 12. La Caixa. Recuperado en Julio 2017 de http:// www.arletteurrea.com/images/ archivo/sucesion_en la_empresa_familia_gallo.pdf.

- Gedajlovic, E., Lubatkin, M.H., y Schulze, W.S. (2004). Crossing the threshold from founder management to professional management: A governance perspective. Journal of Management Studies, 41(5), 899-912.

- Gersick, K.E. (Ed.). (1997). Generation to generation: Life cycles of the family business. Massachusetts, Estados Unidos: Harvard Business Press.

- Gomez-Mejia, L.R., Cruz, C., Berrone, P., y De Castro, J. (2011). The bind that ties: Socioemotional wealth preservation in family firms. The Academy of Management Annals, 5(1), 653-707. 
- Gómez-Mejía, L.R., Haynes, K.T., Núñez-Nickel, M., Jacobson, K.J., y Moyano-Fuentes, J. (2007). Socioemotional wealth and business risks in family-controlled firms: Evidence from Spanish olive oil mills. Administrative science quarterly, 52(1), 106-137.

- Gomez-Mejia, L.R., Larraza-Kintana, M., y Makri, M. (2003). The determinants of executive compensation in family-controlled public corporations. Academy of management journal, 46(2), 226-237.

- Hall, A., y Nordqvist, M. (2008). Professional management in family businesses: Toward an extended understanding. Family Business Review, 21(1), 51-69.

- Hiebl, M.R. (2013). Non-family CFOs in family businesses: do they fit? Journal of Business Strategy, 34(2), 45-51.

- Huybrechts, J., Voordeckers, W., y Lybaert, N. (2012). Entrepreneurial risk taking of private family firms: The influence of a nonfamily CEO and the moderating effect of CEO tenure. Family Business Review, 26(2), 161-179.

- Jorissen, A., Leveren, E., Martens, R., y Reheul, A. M. (2005). Real versus sample-based differences in comparative family business research. Family Business Review, 18(3), 229-246.

- Khurana, R., y Nohria, N. (2000, 08 de mayo). The performance consequences of CEO turnover. Recuperado en Julio 2017 de SSRN: https://ssrn. com/abstract=219129. D0l: http:// dx.doi.org/10.2139/ssrn.219129

- Klein, S., y Bell, F.A. (2007). Nonfamily executives in family businesses: A literature review. Electronic Journal of Family Business Studies (EJFBS), 1(1), 19-37.
- Klein, S.B. (2000). Family businesses in Germany: Significance and structure. Family Business Review, 13(3), 157-181.

- KPGM. (2014). European Family Business Barometer. European Family Businesses. Recuperado en Julio 2017 de http://www.europeanfamilybusinesses.eu/uploads/ Modules/Publications/2d-efb-kpmgfamily-business-barometer_onlineversion.pdf

- Le Breton-Miller I., Miller D., y Steier L.P. (2004). Toward and integrative model of effective $\mathrm{FOB}$ succession. Entrepreneurship theory and practice, 28(04), 305-328.

- Lutz, E., y Schraml, S. (2011). Family firms: Should they hire an outside CFO? Journal of Business Strategy, 33(1), 39-44.

- McConaughy, D.L. (2000). Family CEOs vs. Nonfamily CEOs in the Family Controlled Firm: An Examination of the Level and Sensitivity of Pay to Performance. Family Business Review, 13(2), 121-131.

- Miller, D., Breton-Miller, L., Minichilli, A., Corbetta, G., y Pittino, D. (2014). When do Non Family CEOS Outperform in Family Firms? Agency and Behavioural Agency Perspectives. Journal of Management Studies, 51(4), 547-572.

- Miller, D., y Breton-Miller, L. (2006). Family governance and firm performance: Agency, stewardship, and capabilities. Family business review, 19(1), 73-87.

- Miller, D., Steier, L., y Breton-Miller, I. (2003). Lost in time: intergenerational succession, change, and failure in family business. Journal of business venturing, 18(4), 513-531.

- Ossorio, M. (2011). La figura del non family Ceo nel governo e nella gestione dell'impresa familiare. En M. Vicenzo y M. Del Giudice, Affari di famiglia. Problematiche gestionali e modelli imprenditoriali emergent nei nuovi family business. Milano: Egea. - Perry, J.T., Ring, J.K., y Broberg, J.C. (2014). Which Type of Advisors Do Family Businesses Trust Most? An Exploratory Application of Socioemotional Selectivity Theory. Family Business Review, 28(3), 211-226.

- Rodriguez Ariza, L., Watkins, K., y Briano Turrent, G. (2015). Efectos de la Capacidad y Procedencia de los CEOs en el Desempeño Financiero de las Empresas Cotizadas Familiares: Evidencias para México. Premio Adalberto Viesca Sada 2015. Monterrey. Mexico.

- Sciascia, S., y Mazzola, P. (2008). Family Involvement in Ownnership and Management: Exploring Nonlinear Effects on Performance. Family Business Review, 26(4), 331-345.

- Sciascia, S., Mazzola, P., Chirico, F., y Sirmon, D. G. (2011). Resource orchestration in family firms: Investigating how entrepreneurial orientation, generational involvement, and participative strategy affect performance. Strategic Entrepreneurship Journal, 5(4), 307-326.

- Sonfield, M.C., y Lussier, R.N. (2009). Non-family-members in the family business management team: a multinational investigation. International entrepreneurship and management journal, 5(4), 395-415

- Songini, L., y Vola, P. (2015). The Role of Professionalization and Managerialization in Family Business Succession. Management Control, 1, 9-43. 
- Vandekerkhof, P., Steijvers, T., Hendriks, W., y Voordeckers, W. (2014). The effect of organizational characteristics on the appointment of nonfamily managers in private family firms: The moderating role of socioemotional wealth. Family Business Review, 28(2), 104-122.

- Von Schultzendorff, D. (1984). Fremdmanager im Familienunternehmen: eine empirische Analyse. Maus.
- Ward, J., y Aronoff, C.E. (1994). Managing family-business conflict. Nations Business, 82(11), 54.

- Ward, J.L. (1997). Growing the family business: Special challenges and best practice. Family Business Review, 10(4), 323-337.

(2004). Perpetuating the family business: 50 lessons learned from long lasting, successful families in business. Basingstoke: Palgrave Macmillan.
- Yin, R.K. (1994). Applications of case study research. Applied social research methods series. London and New Delhi: Sage Publications.

(2014). Case study research: Design and methods. Londres: Sage publications. 